\title{
The role of ATP-sensitive potassium channel on acute urinary retention and subsequent catheterization in the rat
}

\author{
Fumiya Ohmasa, Motoaki Saito*, Shogo Shimizu, Sousuke Taniguchi, \\ Fotios Dimitriadis, Itaru Satoh, Yukako Kinoshita and Keisuke Satoh
}

Division of Molecular Pharmacology, Department of Pathophysiological and Therapeutic Science, Tottori University Faculty of Medicine, 86 Nishi-machi, Yonago, 683-8503, Japan

Correspondence:

* Motoaki Saito, MD, PhD

Division of Molecular Pharmacology, Department of Pathophysiological and Therapeutic Science, Tottori University Faculty of Medicine, 86 Nishi-machi, Yonago 683-8503, Japan

$$
\begin{array}{ll}
\text { Telephone: } \quad+81-859-38-6162 \\
\text { FAX: } \quad+81-859-38-6160 \\
\text { E-mail address: } & \text { saitomo@med.tottori-u.ac.jp }
\end{array}
$$




\section{ABSTRACT}

We investigated the role of $\mathrm{K}_{\mathrm{ATP}}$ channel on acute urinary retention (AUR) induced bladder dysfunction. Eight-week-old female Sprague-Dawley rats were divided into seven groups: a sham-operated control group, an AUR group, and five AUR groups treated with: two different $\mathrm{K}_{\mathrm{ATP}}$ channel openers namely nicorandil (3 or $10 \mathrm{mg} / \mathrm{kg}$ ), or cromakalim (100 or $300 \mu \mathrm{g} / \mathrm{kg}$ ), or one $\mathrm{K}_{\text {ATP }}$ channel inhibitor namely glibenclamide (5mg/kg). The drugs were administered 30min before induction of AUR. After the urethra was obstructed with a clip, AUR was induced by intravesical infusion of $2.5 \mathrm{~mL}$ of saline via cystostomy. Following a 30min obstruction the bladder was allowed to drain with a catheter in place for 60min with real-time monitoring of intravesical pressure and blood flow. After the experimental period, the bladder function was assessed, using organ bath techniques (carbachol and 100mM KCl). AUR increased the intravesical pressure and decreased the blood flow. The subsequent catheterization decreased the intravesical pressure and increased the blood flow. AUR group reduced significantly the contractile responses to both carbachol and $\mathrm{KCl}$ compared with the control group.

Nicorandil and cromakalim but not glibenclamide prevented the bladder dysfunction after AUR suggesting that $\mathrm{K}_{\text {ATP }}$ channel openers may prevent the bladder dysfunction caused by AUR and subsequent catheterization.

Keywords: acute urinary retention, nicorandil, cromakalim, glibenclamide, pharmacological preconditioning 


\section{Introduction}

Clinically, acute urinary retention (AUR) is often observed in patients with benign prostate hyperplasia (Meigs et al, 1999), diabetic autonomic neuropathy (Ewing and Clarke, 1982) or as a drug-related side effect (Verhamme et al, 2008). AUR is a significant public health issue with significant consequences to the affected individual (Choong and Emberton, 2000). Traditionally, prolonged bladder overdistension has been thought to simply produce stretch damage of the detrusor muscle and other bladder microstructures, resulting in reduced contractility of the detrusor muscle. Several reports have indicated that the biochemical and metabolic status of the bladder is altered after prolonged overdistension (Tammela et al, 1993; Gabella et al, 1999; Saito et al, 2010). Previous studies from this and other laboratories have demonstrated that ischemia/reperfusion injury might be at least partly responsible for the prolonged bladder dysfunction occurring after overdistension (Lin et al, 2000; Saito and Miyagawa, 2001). Both in humans and animals, acute bladder overdistension induces a reduction in blood flow to bladder tissue resulting in partial ischemia and hypoxia; after subsequent catheterization, the blood supply recovers, allowing reperfusion (Lin et al, 2000; Saito and Miyagawa, 2001). As with ischemia/reperfusion-injury of other organs, ischemia followed by subsequent reperfusion in the bladder leads to oxidative stress, inflammation, and neutrophil infiltration, which might contribute to the delayed recovery of the bladder function. Recently, we reported that treatment with edaravone, a radical scavenger or sivelestat, a neutrophil elastase inhibitor are able to prevent the bladder dysfunction induced by rat ischemia/reperfusion or AUR models (Kono et al, 2008; Shimizu et al, 2009).

Noma (1983) originally described the ATP-sensitive potassium channel (K $\mathrm{K}_{\mathrm{ATP}}$ 
channel) in guinea pig cardiac membrane patches (Noma, 1983). Since then, $\mathrm{K}_{\mathrm{ATP}}$ channels have been detected in several kinds of tissues and they are localized in the sarcolemmal, mitochondrial and nuclear membranes (sarco $\mathrm{K}_{\mathrm{ATP}}$ channels, mito $\mathrm{K}_{\mathrm{ATP}}$ channels, and nuclear $\mathrm{K}_{\mathrm{ATP}}$ channels, respectively) (Zhuo et al, 2005). $\mathrm{K}_{\mathrm{ATP}}$ channels play an important role in ischemia/reperfusion-injury in many tissues. It was found that ischemia leads to cardiac cell apoptosis, which contributes to the injury while preconditioning prevents apoptosis in ischemic hearts. Opening of $\mathrm{K}_{\mathrm{ATP}}$ channels can protect cardiac myocytes against ischemia/reperfusion -injury, called pharmacological preconditioning (Zhuo et al, 2005). There are some reports indicating that nicorandil is a

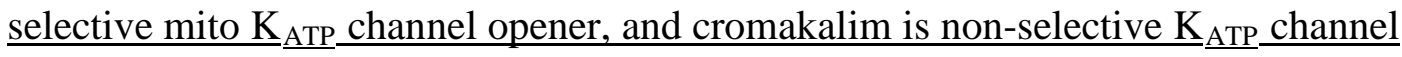
opener whereas glibenclamide is a non-selective $\mathrm{K}_{\text {ATP }}$ channel inhibitor (Date et al, 2005).

Therefore, we hypothesized that $\mathrm{K}_{\text {ATP }}$ channel openers might prevent the bladder dysfunction caused by the AUR and subsequent catheterization. In the current international literature there is no information available regarding the effects of $\mathrm{K}_{\mathrm{ATP}}$ channel openers or inhibitors on bladder dysfunction following AUR and subsequent catheterization. Thus the aim of the present study was to investigate the effects of $\mathrm{K}_{\mathrm{ATP}}$ channel openers, nicorandil and cromakalim, and $\mathrm{K}_{\mathrm{ATP}}$ channel inhibitor, glibenclamide in the bladder dysfunction after AUR and subsequent catheterization. 


\section{Materials and methods}

\subsection{Animals and experimental design}

All animal experiments were conducted in accordance with the guidelines set by the Tottori University Committee for Animal Experimentation. The rat experimental model was created as described in our previous reports (Saito and Miyagawa, 2001; Shimizu et al, 2009). Eight-week-old female Sprague-Dawley rats (160-200g; SLC, Shizuoka, Japan) were randomly divided into seven groups ( $n=5-6$ in each group). Group A consisted of sham-operated control rats, and groups B, C, D, E, F and G undergone 30 min of overdistension followed by 60 min of drainage; group B was non-drug treated; groups C and D were treated with nicorandil at a dose of 3 or $10 \mathrm{mg} / \mathrm{kg}$ body weight, respectively; groups $\mathrm{E}$ and F were treated with cromakalim at a dose of 100 or $300 \mu \mathrm{g} / \mathrm{kg}$ body weight, respectively; and group $\mathrm{G}$ was treated with glibenclamide $5 \mathrm{mg} / \mathrm{kg}$ body weight. Each drug was administrated intra-abdominally 30min before inducing AUR. Each rat was anaesthetized with sodium pentobarbital (50mg/kg, intra-abdominally). The rat distal urethra was then clamped with a small clip; subsequently a cystostomy was made and $2.5 \underline{\mathrm{ml}}(0.5 \underline{\mathrm{ml}} / \mathrm{min})$ of saline was infused with an infusion pump (TOP-5200; TOP, Tokyo, Japan) to induce AUR. Thirty minutes after inducing urinary retention, the cystostomy tube was opened and the bladder was allowed to empty for another 60 min. The rats were euthanized with an overdose of pentobarbital $(60 \mathrm{mg} / \mathrm{kg}$, intra-abdominally).

\subsection{Simultaneous measurements of intravesical pressure and blood flow in the bladder}

A cystometry catheter was connected to an external pressure transducer (P2310; 
Gould, Eastlake, $\mathrm{OH}$ ) for the measurement of the intravesical pressure. Simultaneously, the blood flow in the rat bladder was measured in vivo with a laser Doppler flow meter (BRL-100; Bioresearch Co., Nagoya, Japan) according to the method we described in our previous reports (Saito and Miyagawa, 2001; Shimizu et al, 2009).

\subsection{Tissue preparation and measurement of contractile force of the bladder}

Functional studies were conducted according to the methods described in our previous reports (Saito et al, 2008, 2010; Shimizu et al, 2009). Briefly, longitudinal strips of the posterior wall of the bladder dome were mounted in organ baths ( $25 \underline{\mathrm{ml}}$ ) containing Krebs-Henseleit solution, and bubbled with $5 \% \mathrm{CO}_{2}$ and $95 \% \mathrm{O}_{2}\left(37^{\circ} \mathrm{C}\right)$. One hook was suspended from a transducer (type 45196 A; San-ei Instruments, Tokyo, Japan), and the lower hook was fixed to a plastic support leg attached to a micrometer (Mitutoyo, Tokyo, Japan). Each strip was equilibrated unstretched for $30 \mathrm{~min}$. A load of $1.0 \mathrm{~g}$ was applied to each strip by micrometer adjustment, and the load was readjusted to this level $30 \mathrm{~min}$ later. Changes in the tone of the strips were measured isometrically using force transducers, and the data were recorded on a personal computer (Macintosh G3; Apple Computer, Cupertino, CA) using the Chart v3.6.9 software and the PowerLab/16sp data acquisition system (AD Instruments, Castle Hill, Australia). Cumulative concentration response curves to carbachol were constructed, and contractile forces to $100 \mathrm{mM}$ of KCl were also measured.

\subsection{Data analysis}

$\underline{\text { The } \mathrm{EC}_{50}} \underline{\text { and }} \mathrm{E}_{\max }$ values to carbachol were determined using a Macintosh computer (G3) loaded with the Chart v3.6.9 software and the Power Lab/16sp data 
acquisition system. Contractile data were calculated as grams of active force per cross-sectional area in square millimeters. The cross-sectional area was calculated by using the following equation: cross-sectional area = weight/ (length $\times 1.05$ ), where 1.05 is the assumed density of the smooth muscle (Saito et al, 2008; Shimizu et al, 2009). A statistical comparison of differences between groups was performed with the use of analysis of variance and Fisher's multiple comparison tests. P values less than 0.05 were considered to be statistically significant.

\subsection{Drugs and chemicals}

Nicorandil was kindly supplied by Chugai Pharmaceutical Co. Ltd (Tokyo, Japan). Cromakalim and glibenclamide were purchased from Sigma-Aldrich (St. Louis, MO). All other chemicals are available commercially. 


\section{Results}

\subsection{Measurement of intravesical pressure and blood flow in the rat bladder during urinary retention and subsequent catheterization}

Figure 1 shows the result of the simultaneous measurement of intravesical pressure and blood flow in the rat bladder during urinary retention and subsequent catheterization. In all the experimental groups, urinary retention increased the intravesical pressure and decreased blood flow, and subsequent catheterization decreased intravesical pressure and increased blood flow. Treatment with nicorandil or cromakalim tended to increase the blood flow during the catheterization phase in a dose dependent fashion, and treatment with glibenclamide decreased the blood flow during the catheterization phase compared to that in group B. At the end of the experiments ( 95 minutes since induction of AUR), $p$ values of group B vs groups C, D, E, F and G in the blood flow were $0.308,0.028,0.812$, $\underline{0.395}$ and 0.180 , respectively. On the other hand, there were no significant differences in the intravesical pressure of the bladder between any of the other groups during the experimental period. These data suggest that the treatment with $\mathrm{K}_{\mathrm{ATP}}$ channel openers or $\mathrm{K}_{\text {AтP }}$ channel inhibitor did not alter the intravesical pressure during AUR and subsequent catheterization, and that the treatment with $\mathrm{K}_{\text {ATP }}$ channel openers significantly increased the blood flow during the catheterization phase.

\subsection{Measurement of contractile response to carbachol and $100 \mathrm{mM} \mathrm{KCl}$}

The $\mathrm{E}_{\max }$ and $\mathrm{EC}_{50}$ values for the contractile response of bladder strips to carbachol and the response to $\mathrm{KCl}(100 \mathrm{mM})$ were determined, and the results are shown in Table 1. The $E_{\max }$ values of carbachol in the $\underline{\mathrm{AUR}}$ rats (group B) were markedly smaller than those of the control rats (group A). Treatment with nicorandil and cromakalim $\mathrm{K}_{\mathrm{ATP}}$ channel 
openers (group C-F) significantly prevented this injury. On the other hand, treatment with the $\mathrm{K}_{\mathrm{ATP}}$ channels inhibitor glibenclamide failed to prevent the bladder smooth muscle dysfunction (group $\mathrm{G}$ ). The $\mathrm{EC}_{50}$ values of the group B were significantly greater than those of the control group (group A). However, a part from the significant difference between groups A and B, there were no statically significant differences between any of the other groups (Table 1). Contractile responses to $100 \mathrm{mM} \mathrm{KCl}$ showed a similar patter to that for the Emax values of carbachol in each group. 


\section{Discussion}

In the present study, we clearly demonstrated that during the AUR phase the urinary bladder was under ischemia and that during and after drainage the bladder was under reperfusion. Interestingly, although in the bladder blood flow there were no significant differences between any of the groups during AUR period, treatment with nicorandil or cromakalim increased the blood flow during the catheterization phase in a dose-dependent manner compared to that in the non-drug treated AUR animals. On the other hand, treatment with glibenclamide decreased the blood flow during the catheterization phase compared to that in the non-drug treated AUR animals. These phenomena may be explained by the action of the $\mathrm{K}_{\mathrm{ATP}}$ channel openers. In fact, it is possible that the activation of the $\mathrm{K}_{\text {ATP }}$ channels by the $\mathrm{K}_{\text {ATP }}$ channel openers might have resulted in dilated vessels so that blood flow increased during the drainage phase. In $\underline{\text { addition, as nicorandil is reported to have properties as both } \mathrm{K}_{\text {ATP }} \text { channel opener and }}$ nitric oxide (NO) donor (Kamiyama et al, 2007), it is possible that NO directly dilated the vessels in the bladder. Another possible mechanism could be that $\mathrm{K}_{\text {ATP }}$ channel openers directly relax the bladder smooth muscle and subsequently the blood flow increase during the drainage phase.

Gopalakrishnan and associates (1999) provided evidence for the molecular identity of $\mathrm{K}_{\mathrm{ATP}}$ channels expressed in guinea pig bladder smooth muscle cells. Employing pharmacological and molecular analysis, they demonstrated that the $\mathrm{K}_{\text {ATP }}$ channels in the bladder smooth muscle cells are composed of SUR2B associated with a single inward $\underline{\text { rectifier, } K_{\underline{I R}}} \underline{6.2 .}$ (Gopalakrishnan et al, 1999). Fabiyi and co-workers (2003) reported that, $\mathrm{K}_{\mathrm{ATP}}$ channel openers inhibit both neurogenic and myogenic bladder contraction (Fabiyi et al 2003). Since then some investigators have reported the inhibitory effects of 
$\mathrm{K}_{\text {ATP }}$ channel openers on bladder contractility for the purpose of clinical use in patients with overactive bladder (Kamiyama et al, 2007; Yunoki et al, 2008; Akino et al, 2008). However, there is no information available concerning the role of $\mathrm{K}_{\mathrm{ATP}}$ channels on AUR associated bladder dysfunction as a result of ischemia/reperfusion-injury.

The role of $\mathrm{K}_{\text {АTP }}$ channels in cardiovascular system has been studied adequately and their most prominent function is that the opening of these channels can protect cardiac myocytes against ischemic injury. More specifically sarco $\mathrm{K}_{\mathrm{ATP}}$ channels are reported to regulate the cell energy metabolism in two ways: a) one is that opening of sarco $\mathrm{K}_{\text {AтP }}$ channel accelerates phase 3 repolarization and slows down depolization, which will shorten action potential duration and prevent the reversal of $\mathrm{Na}^{+} / \mathrm{Ca}^{2+}$ exchanger resulting in inhibition of $\mathrm{Ca}^{+2}$ entry in the cell and b) the second way is induction of membrane potential hyperpolarization (Zhuo et al, 2005). On the other hand in case of energy crisis, such as in an ischemia/reperfusion-injury the mitochondrial production of ATP is impaired by alternation of mitochondrial membrane potential, imbalanced trans-membrane ions transport, or over-production of free radicals. The opening of mito $\mathrm{K}_{\mathrm{ATP}}$ channels prevent the mitochondria from these disturbances by controlling the mitochondrial $\mathrm{Ca}^{2+}$ ion concentration and matrix swelling (Zhuo et al, 2005).

$\underline{\text { Previously we clearly demonstrated that mechanical preconditioning prevents }}$ ischemia/reperfusion induced bladder dysfunction in the rat (Hisadme et al, 2007). In order to investigate the effect of pharmacological preconditioning on AUR-induced bladder dysfunction, we used three drugs namely nicorandil, cromakalim and glibenclamide. Nicorandil is reported to be a selective mito $\mathrm{K}_{\text {ATP }}$ channel opener, and cromakalim is reported to be a non-selective $\mathrm{K}_{\text {ATP }}$ channel opener whereas glibenclamide 


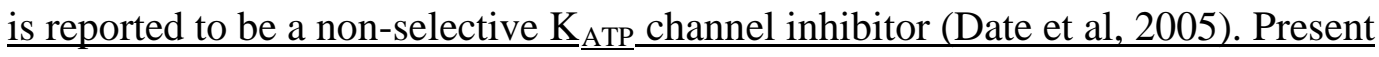
functional studies showed that nicorandil and cromakalim but not glibenclamide prevented the bladder dysfunction after AUR. Our data indicate that pharmacological preconditioning protects the bladder smooth muscle against ischemic (and reperfusion) insult, and that this effect is mediated via the opening of the mito $\mathrm{K}_{\text {ATP }}$ channels. However, there are still controversies about the existence of the mito $\mathrm{K}_{\underline{A T P}}$ channels in the mitochondria (Zhuo et al, 2005). Further investigation is required to clarify the underlying molecular mechanisms of $\mathrm{K}_{\text {ATP }}$ channels in preventing AUR-induced bladder dysfunction.

In conclusion, $\mathrm{K}_{\mathrm{ATP}}$ channel openers prevented the bladder dysfunction caused by AUR and subsequent catheterization.

\section{Acknowledgements}

This study was supported by a grant in aid from the Ministry of Education, Science, and Culture of Japan (\#20591880). 


\section{Refernces}

Akino, H., Chapple, C.R,. McKay, N., Cross, R.L., Murakami, S., Yokoyama, O., Chess-Williams, R., Sellers, D.J., 2008. Spontaneous contractions of the pig urinary bladder: the effect of ATP-sensitive potassium channels and the role of the mucosa. BJU Int. 102, 1168-1174.

Choong, S., Emberton, E., 2003. Acute urinary retention. BJU Int. 85, 186-201.

Date, T., Taniguchi, I., Inada, K., Matsuo, S., Miyanaga, S., Yamane, T., Ab,e Y., Sugimoto, K., Mochizuki, S., 2005. Nicorandil inhibits serum starvation-induced apoptosis in vascular endothelial cells. J Cardiovasc. Pharmacol. 46, 721-726.

Ewing, D.J., Clarke B,F., 1982. Diagnosis and management of diabetic autonomic neuropathy. Br. Med. J. (Clin Res Ed). 2, 285: 916-8.

Fabiyi, A.C,, Gopalakrishnan, M., Lynch, J.J. 3rd, Brioni, J.D., Coghlan, M.J., Brune, M.E., 2003. In vivo evaluation of the potency and bladder-vascular selectivity of the ATP-sensitive potassium channel openers (-)-cromakalim, ZD6169 and WAY-133537 in rats. BJU Int. 91, 284-90.

Gabella, G., Uvelius, B., 1999. Structural changes in the rat bladder after acute outlet obstruction. Scand. J. Urol. Nephrol. 201, 32-37.

Gopalakrishnan, M., Whiteaker, K.L., Molinari, E.J., Davis-Taber, R., Scott, V.E., Shieh, 
C.C., Buckner, S.A., Milicic, I., Cain, J.C., Postl, S., Sullivan, J.P., Brioni, J.D., 1999. Characterization of the ATP-sensitive potassium channels $\left(\mathrm{K}_{\mathrm{ATP}}\right)$ expressed in guinea pig bladder smooth muscle cells. J. Pharmacol. Exp. Ther. 289, 551-558.

Hisadome, Y., Saito, M., Kono, T., Satoh, I., Kinoshita, Y., Satoh, K., 2007. Beneficial effect of preconditioning on ischemia-reperfusion injury in the rat bladder in vivo. Life Sci. 81, 347-352.

Kamiyama, Y., Muto, S., Masuda, H., Ide, H., Ishizuka, N., Saito, K., Horie, S., 2008. Inhibitory effects of nicorandil, a K ATP channel opener and a nitric oxide donor, on overactive bladder in animal models. BJU Int. 101,360-365.

Kono, T., Okada, S., Saito, M., 2008. Neutrophil elastase inhibitor, sivelestat sodium hydrate prevents ischemia-reperfusion injury in the rat bladder. Mol. Cell. Biochem. 311, 87-92.

Lin, A.T., Chen, K.K., Yang, C.H., Chang, L.S., 2000. Mannitol facilitates rabbit urinary bladder recovery from overdistension injury. Urology 56, 702-707.

Meigs, J.B., Barry M,J., Giovannucci, E., Rimm, E.B,, Stampfer, M.J., Kawachi, I. , 1999. Incidence rates and risk factors for acute urinary retention: the health professionals followup study. J Urol. 162, 376-82.

Noma, A,. 1983. ATP-regulated K+ channels in cardiac muscle. Nature 305, 147-148. 
Saito, M., Miyagawa, I., 2001. Bladder dysfunction after acute urinary retention in rats. J. Urol. 165, 1745-1747.

Saito, M., Okada, S., Kazuyama, E., Satoh, I., Kinoshita, Y., Satoh, K., 2008. Pharmacological properties, functional alterations, and gene expression of muscarinic receptors in young and old type 2 Goto-Kakizaki diabetic rat bladders. J. Urol. 180, 2701-2705.

Saito, M., Shimizu, S, Kinoshita, Y., Satoh, I., Shomori, K., Dimitriadis, F., Satoh, K., 2010. Bladder dysfunction after acute urinary retention in the rats: a novel over active bladder model. Mol. Cell. Biochem. 333, 109-114.

Shimizu, S., Saito, M., Kinoshita, Y., Kazuyama, E., Tamamura, M., Satoh, Satoh, K., 2990. Acute urinary retention and subsequent catheterization cause lipid peroxidation and oxidative DNA damage in the bladder; preventive effect of edaravone, a free radical scavenger. BJU Int. 104, 713-717.

Tammela, T.L., Levin, R.M., Monson, F.C., Wein, A.J., Longhurst, P.A., 1993. The influence of acute overdistension on rat bladder function and DNA synthesis. J. Urol. 150, 1533-1539.

Verhamme, K.M., Sturkenboom, M.C., Stricker, B.H., Bosch, R., 2008. Drug-induced urinary retention: incidence, management and prevention. Drug Saf. 31, 373-88. 


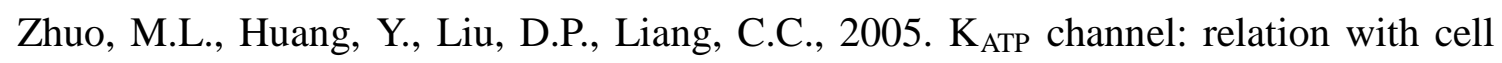
metabolism and role in the cardiovascular system. Int. J. Biochem. Cell Biol. 37, 751-64.

Yunoki, T., Zhu, H.L., Iwasa, K., Tomoda, T., Aishima, M., Shibata, A., Naito, S., Teramoto, N., 2008. Comparative studies of ZD0947, a novel ATP-sensitive $\mathrm{K}(+)$ channel opener, on guinea pig detrusor and aortic smooth muscles. Naunyn. Schmiedeberg. Arch. Pharmacol. 376, 309-319. 


\section{Figure legends}

Intravesical pressure and blood flow measured simultaneously during experimental duration.

Nic 3: AUR rats treated with nicorandil at $3 \mathrm{mg} / \mathrm{kg}$ body weight, Nic10: AUR rats treated with nicorandil at $10 \mathrm{mg} / \mathrm{kg}$ body weight, Cro 100: AUR rats treated with cromakalim at $100 \mu \mathrm{g} / \mathrm{kg}$ body weight, Cro 300: AUR rats treated with cromakalim at $300 \mu \mathrm{g} / \mathrm{kg}$ body weight, and Glib 5: AUR rats treated with glibenclamide $5 \mathrm{mg} / \mathrm{kg}$ body weight. 


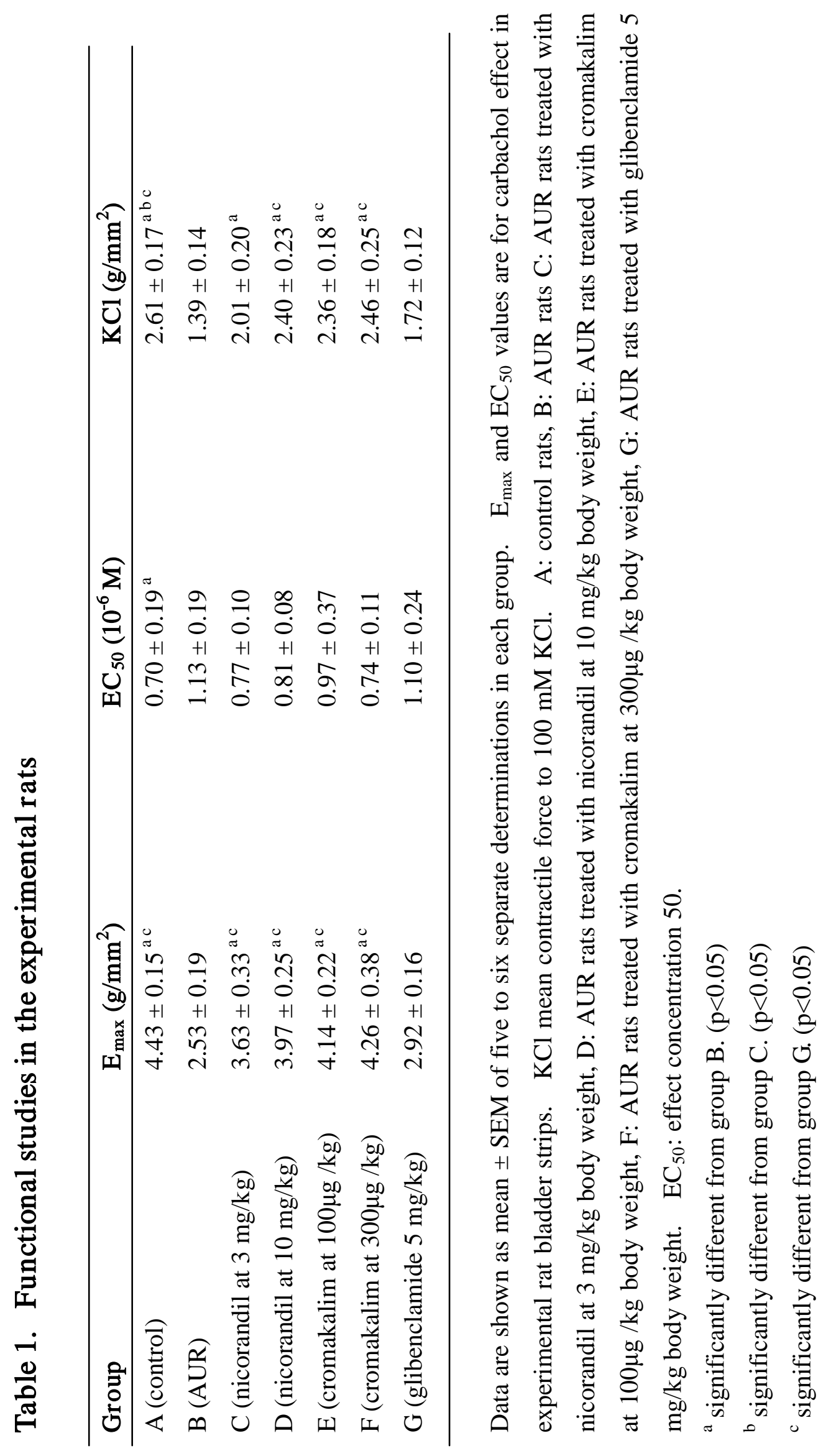



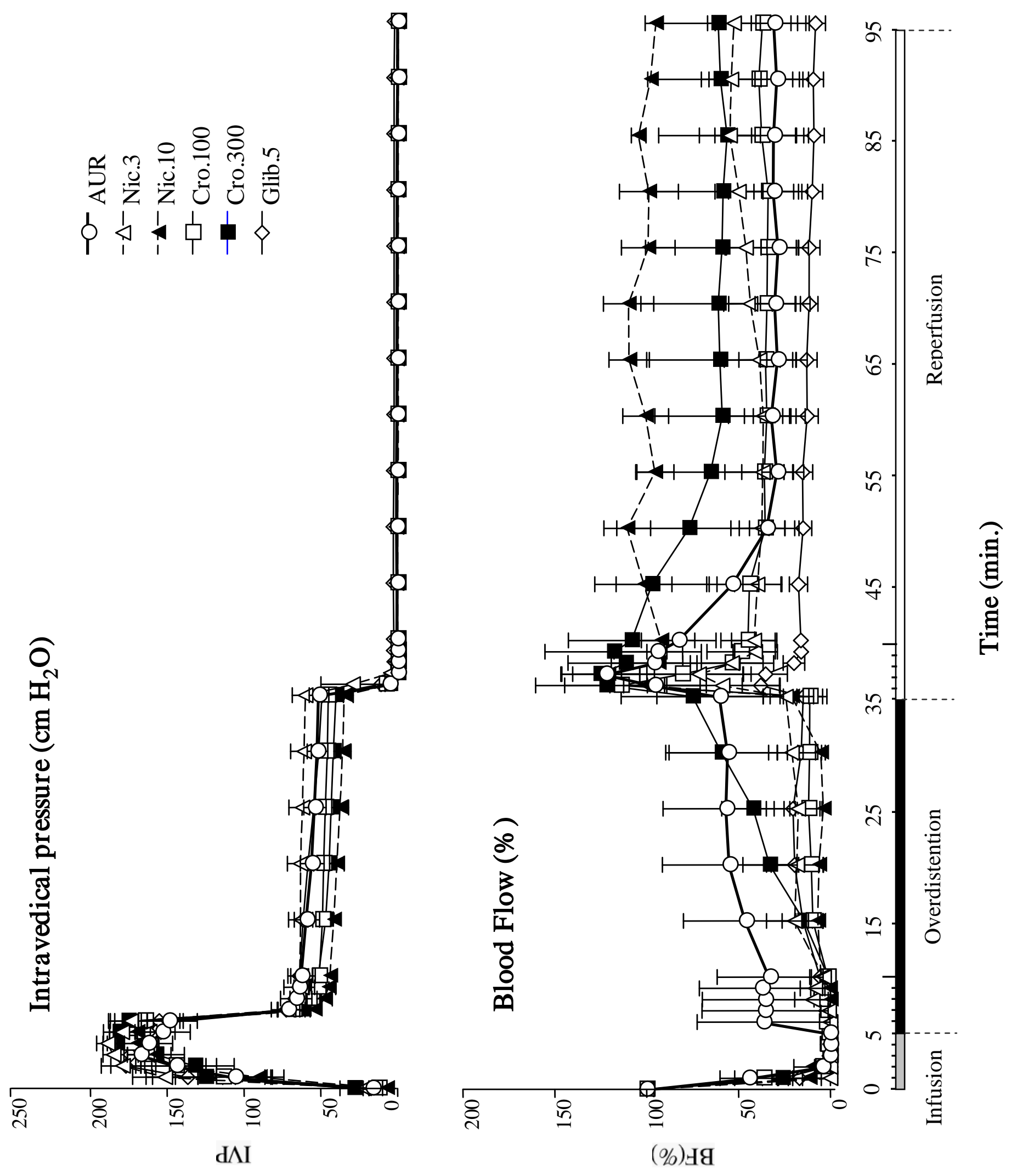